\title{
Fahr's Syndrome
}

National Institute of Neurological Disorders and Stroke (NINDS)

\section{Source}

National Institute of Neurological Disorders and Stroke (NINDS). Fahr's Syndrome

Information Page.

Fahr's Syndrome is a rare, genetically dominant, inherited neurological disorder characterized by abnormal deposits of calcium in areas of the brain that control movement, including the basal gang lia and the cerebral cortex. Symptoms of the disorder may include deterioration of motor function, dementia, seizures, headache, dysarthria (poorly articulated speech), spasticity (stiffness of the limbs) and spastic paralysis, eye impairments, and athetosis (involuntary, writhing movements). Fahr's Syndrome can also include symptoms characteristic of Parkinson's disease such as tremors, muscle rigidity, a mask-like facial appearance, shuffling gait, and a "pill-rolling" motion of the fingers. These symptoms generally occur later in the development of the disease. More common symptoms include dystonia (disordered muscle tone) and chorea (involuntary, rapid, jerky movements). Age of onset is typically in the 40s or 50s, although it can occur at any time in childhood or adolescence. 